\title{
NudC Associates with Lis1 and the Dynein Motor at the Leading Pole of Neurons
}

\author{
Jonathan P. Aumais, ${ }^{1}$ James R. Tunstead, ${ }^{3,4}$ Robert S. McNeil, ${ }^{2}$ Bruce T. Schaar, ${ }^{,}$Susan K. McConnell, ${ }^{5}$ \\ Sue-Hwa Lin, ${ }^{4}$ Gary D. Clark, ${ }^{2}$ and Li-yuan Yu-Lee ${ }^{1,3}$ \\ Departments of ${ }^{1}$ Molecular and Cellular Biology, ${ }^{2}$ Pediatrics, Neurology and Neuroscience, and ${ }^{3}$ Medicine and \\ Immunology, Baylor College of Medicine, and ${ }^{4}$ Department of Molecular Pathology, M. D. Anderson Cancer Center, \\ Houston, Texas 77030, and ${ }^{5}$ Department of Biological Sciences, Stanford University, Stanford, California 94305
}

NUDC is a highly conserved protein important for nuclear migration and viability in Aspergillus nidulans. Mammalian NudC interacts with Lis1, a neuronal migration protein important during neocorticogenesis, suggesting a conserved mechanism of nuclear movement in $A$. nidulans and neuronal migration in the developing mammalian brain (S. M. Morris et al., 1998). To further investigate this possibility, we show for the first time that NudC, Lis1, and cytoplasmic dynein intermediate chain (CDIC) colocalize at the microtubule organizing center (MTOC) around the nucleus in a polarized manner facing the leading pole of cerebellar granule cells with a migratory morphology. In neurons with stationary morphology, NudC is distributed throughout the soma and colocalizes with CDIC and tubulin in neurites as well as at the MTOC. At the subcellular level, NudC, CDIC, and p150 dynactin colocalize to the interphase microtubule array and the MTOC in fibroblasts. The observed colocalization is confirmed biochemically by coimmunoprecipitation of NudC with CDIC and cytoplasmic dynein heavy chain (CDHC) from mouse brain extracts. Consistent with its expression in individual neurons, a high level of NudC is detected in regions of the embryonic neocortex undergoing extensive neurogenesis as well as neuronal migration. These data suggest a biochemical and functional interaction of NudC with Lis1 and the dynein motor complex during neuronal migration in vivo.

Key words: NudC; Lis1; dynein; dynactin; centrosome; neuronal migration
$N u d C$ was first identified as a nuclear distribution (nud) gene that regulates nuclear movement in the filamentous fungus Aspergillus nidulans (Osmani et al., 1990; Morris, 2000). In Aspergillus, nudC is required for nuclear distribution, cell wall deposition, colony growth, and viability (Osmani et al., 1990; Chiu et al., 1997), and it functions, at least in part, by regulating the levels of another nud gene product, NUDF. Mutations in a single allele of the human $n u d F$ homolog, LIS1, result in lissencephaly, a severe malformation of the cerebral cortex (Reiner et al., 1993). Lissencephaly patients display aberrant cortical lamination and lack the gyri and sulci evident in the normal adult brain. Genetic studies in both human and mouse show that Lis1 is required for migration of neuronal progenitors through the embryonic intermediate zone (IZ) to the cortical plate (CP) (Wynshaw-Boris and Gambello, 2001). Lis1 may also be involved in the commitment of neuronal progenitors by influencing cleavage orientation in the embryonic ventricular zone (VZ) (Faulkner et al., 2000). The structure of Lis1, a coiled-coil domain followed by seven WD40 $\beta$-transducin-like repeats, suggests that it may be involved in

\footnotetext{
Received May 11, 2001; revised Aug. 27, 2001; accepted Sept. 12, 2001.

This work was supported by American Cancer Society Grant DDC-88885 (B.S.), National Institutes of Health (NIH) Grants MH51864 and HFSP RG0283 (S.K.M.), Cain Foundation Laboratories, NIH Grants NS37146 and NS38289 (G.D.C.), NIH Grant CA64856 (S.H.L.), and NIH Grant DK53176 (L.Y.L.). We acknowledge the Huffington Center on Aging and Dr. Farrah Kheradmand for use of fluorescence microscope, and Drs. Sophia Tsai and Cheng Zhow for brain sections and for critical comments.

J.P.A. and J.R.T. contributed equally to this work.

Correspondence should be addressed to Dr. Li-yuan Yu-Lee, Department of Medicine, Baylor College of Medicine, One Baylor Plaza, Houston, TX 77030. E-mail: yulee@bcm.tmc.edu.

Copyright (ㄷ) 2001 Society for Neuroscience $\quad 0270-6474 / 01 / 210001-07 \$ 15.00 / 0$
}

protein-protein interactions. Lis1 interacts biochemically with several gene products of the nud pathway in mammals, namely cytoplasmic dynein (Faulkner et al., 2000; Smith et al., 2000), mNudE (Efimov and Morris, 2000; Feng et al., 2000), and $\mathrm{mNu}$ deL (Niethammer et al., 2000; Sasaki et al., 2000). Although its precise mechanism of action remains unknown, Lis1 has been shown to regulate dynein motor function (Faulkner et al., 2000; Smith et al., 2000) and is suggested to play a role in neuronal migration (Feng and Walsh, 2001; Vallee et al., 2001; WynshawBoris and Gambello, 2001). We showed previously that NudC forms a complex with Lis1 by coimmunoprecipitation from mouse brain extracts and by yeast two-hybrid and GST pull-down assays (S. M. Morris et al., 1998). We and others have proposed that the NudC/Lis1 complex participates in the development of the mammalian cortex (N. R. Morris et al., 1998; S. M. Morris et al., 1998).

Recent studies colocalized Lis1 and cytoplasmic dynein intermediate chain (CDIC) in the VZ of embryonic day (E) 13 brain and showed high Lis1 expression in the $\mathrm{CP}$ and marginal zone

This article is published in The Journal of Neuroscience, Rapid Communications Section, which publishes brief, peerreviewed papers online, not in print. Rapid Communications are posted online approximately one month earlier than they would appear if printed. They are listed in the Table of Contents of the next open issue of JNeurosci. Cite this article as: JNeurosci, 2001, 21:RC187 (1-7). The publication date is the date of posting online at www.jneurosci.org.

http://www.jneurosci.org/cgi/content/full/5895 

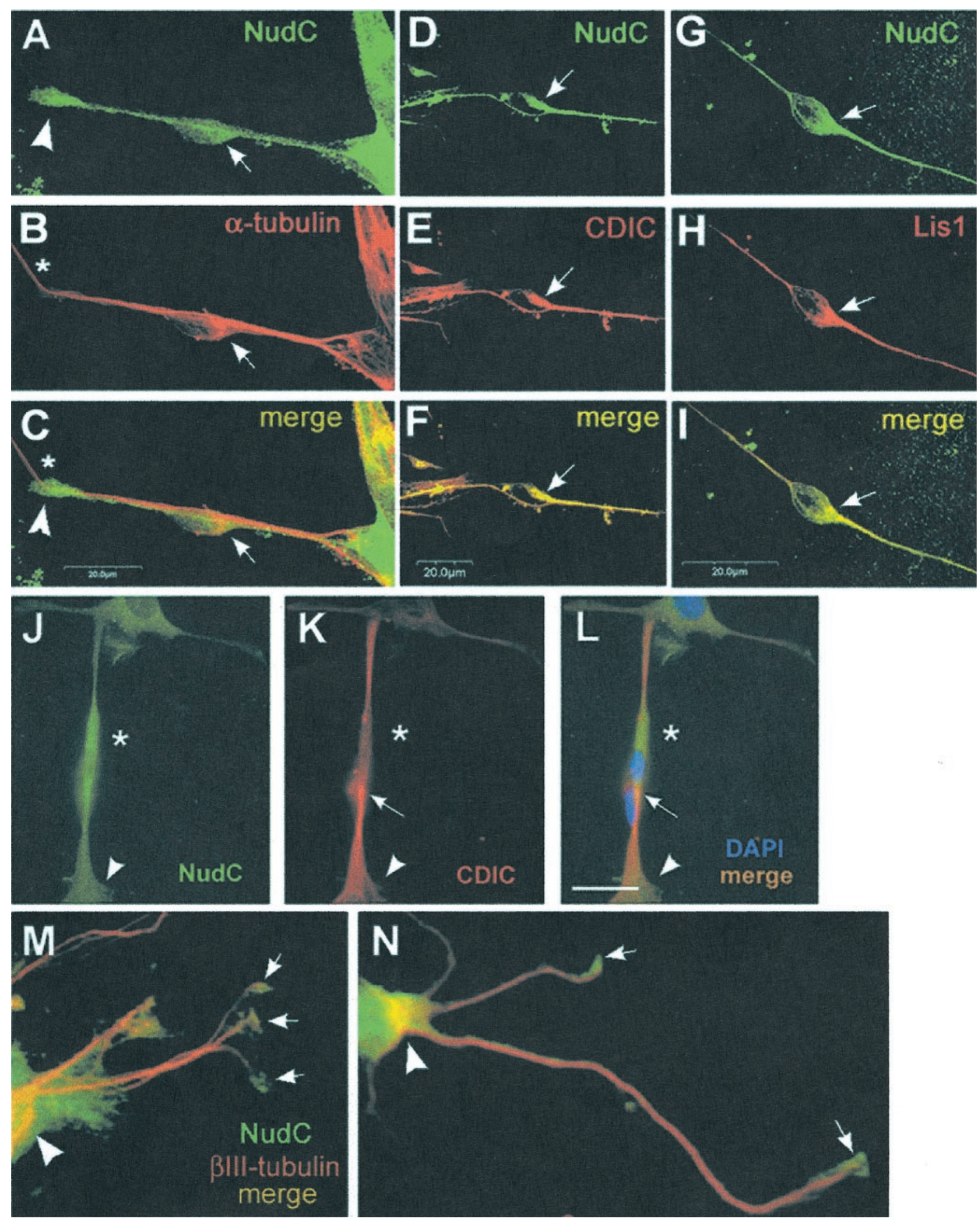

Figure 1. NudC colocalizes with cytoplasmic dynein intermediate chain $(C D I C)$ and Lis1 in cerebellar granule cells and cortical neurons. $A-C$, In neurons prepared from cerebellar granule cell clusters, $\mathrm{NudC}$ (green) immunoreactivity is enriched at the neurite tip (arrowhead) and in the soma rostral to the nucleus (arrow). B, Tubulin immunoreactivity $(\mathrm{red})$ is evident around the nucleus and indicates the position of the MTOC facing the leading pole (arrow). The asterisk indicates the process on which this neuron is migrating. C, Strong overlap (yellow) of NudC and tubulin is detected at the MTOC, which indicates the direction of migration (arrow). $D-F$, NudC colocalizes with CDIC (red) and with $(G-I)$ Lis 1 (red) at the leading pole 
(MZ) of E16 brain (Smith et al., 2000), consistent with a role for these proteins in both the generation and migration of neuronal progenitors in the developing cerebral cortex. Because NudC and Lis1 genes are coexpressed in the $\mathrm{VZ}$ of the forebrain and in the CP at the same embryonic stages (S. M. Morris et al., 1998), we sought to determine whether NudC is also involved in embryonic brain development. We also investigated for the first time the association of NudC with Lis1 and components of the dynein molecular motor in cultured neurons and by coimmunoprecipitation in mouse brain extracts. These studies place NudC in a Lis1/dynein/dynactin complex that suggests a crucial role for the motor complex during corticogenesis and neuronal migration.

\section{MATERIALS AND METHODS}

Cell culture. Cerebellar tissue was obtained from 4-8 d postnatal mice, dissociated, and either plated directly (to obtain neurons with stationary morphology) or allowed to form reaggregated cell clusters (to obtain neurons with migratory morphology) (Bix and Clark, 1998). After 24-36 $\mathrm{h}$ of neurite outgrowth and neuronal migration, cerebellar granule cells were fixed in $4 \%$ paraformaldehyde and processed for antibody labeling. Cortical neurons were isolated from E15 rat embryos, plated at low density onto laminin-coated four-well slides (Becton Dickinson, Mountain View, CA), and cultured in Neurobasal supplemented with B-27, Penn-Strep/glutamine (Life Technologies, Gaithersburg, MD), and 5 $\mathrm{mg} / \mathrm{ml}$ glucose for $2 \mathrm{~d}$ to extend neurites. Mouse embryonic fibroblasts from $129 \mathrm{~Sv}$ mice and COS-1 cells were grown in Opti-MEM II (Life Technologies) with 4\% FBS and plated at low density on poly-D-lysinecoated coverslips (Boehringer Mannheim, Mannheim, Germany).

Immunocytochemistry. Brain sections were deparaffinized, rehydrated, heated to $100^{\circ} \mathrm{C}$ for $20 \mathrm{~min}$ in $10 \mathrm{~mm}$ sodium citrate, $\mathrm{pH} 6$, blocked with horse serum, and incubated with two affinity-purified anti-NudC antibodies, one directed against a $\mathrm{C}$-terminal oligopeptide and the other against a maltose binding protein-NudC fusion protein (MBP-NudC) (Morris and Yu-Lee, 1998). The specificity of NudC staining was shown by staining with secondary antibody alone and by a $2 \mathrm{~h}$ preincubation of anti-NudC with an excess of NudC C-oligopeptide (immunogen) before the antibody was applied to slides. Slides were then incubated with biotinylated secondary antibodies generated against the appropriate species (Vector Laboratories, Burlingame, CA) and visualized by the addition of avidin-FITC conjugate (for NudC) according to manufacturer's protocol. For double-staining experiments, slides were first stained with anti-NudC, blocked with a biotin blocking solution (Vector Laboratories), and incubated with goat anti-Lis1 (a gift of Dr. Gregor Eichele, Baylor College of Medicine), anti- $\alpha$ tubulin (DM1A; Sigma, St. Louis, MO), anti- $\beta$ III tubulin (TuJ1; Babco, Richmond, CA), anti- $\gamma$ tubulin (GTU-88; Sigma), anti-p150 dynactin (150.1; Transduction Laboratories, Lexington, KY), or anti-CDIC antibodies (74.1; Babco). To extract soluble proteins from fibroblasts, cells were treated for $1 \mathrm{~min}$ at $4^{\circ} \mathrm{C}$ in PEM (80 mM K-PIPES, pH 6.8, 5 mM EGTA, pH 7.0, 2 mм $\mathrm{MgCl}_{2}$ ), $0.5 \%$ Triton $\mathrm{X}-100$, and $4 \%(\mathrm{w} / \mathrm{v})$ polyethylene glycol-6000 and fixed at $4^{\circ} \mathrm{C}$ in $\mathrm{PEM} / 4 \%$ formaldehyde for $20 \mathrm{~min}$. Slides were mounted in Vectashield (Vector Laboratories) containing 4',6 diamidino-2phenylindole (DAPI) and photographed using a triple-band (red/green/ blue) emission filter in conjunction with single, double, or triple (yellow, blue, and ultraviolet) excitation bands.

Laser confocal imaging. Confocal images were acquired using a Fluoview FV300 confocal laser scanning unit mounted on a BX50WI fixed stage upright microscope (Olympus America Inc., Melville, NY). Z-series image stacks of 10-11 steps were acquired in 0.7 or $1 \mu \mathrm{m}$ increments. Images were processed using both Fluoview and Adobe Photoshop 5.5 (Mountain View, CA) software.

Coimmunoprecipitation assay. Brains from adult $129 \mathrm{~Sv}$ mice were homogenized in $50 \mathrm{~mm}$ Tris, pH 7.4, $150 \mathrm{~mm} \mathrm{NaCl,} 2 \mathrm{~mm}$ EDTA, $0.1 \%$
NP-40, 2.5\% glycerol, and a protease inhibitor mixture (Sigma). Two milligrams of brain extract were incubated with either $5 \mu \mathrm{g}$ of rabbit immunoglobulin (Vector Laboratories) or $5 \mu \mathrm{l}$ of monoclonal anticytoplasmic dynein heavy chain (CDHC) antibody (440.1; Sigma) that had been preadsorbed to protein G-Sepharose beads (Amersham Pharmacia, Arlington Heights, IL). Antigen-antibody complexes were resolved on a $10 \%$ SDS-PAGE gel, transferred to polyvinylidene difluoride membranes (Bio-Rad, Hercules, CA), and immunoblotted using a mixture of anti-NudC and anti-CDIC antibodies.

\section{RESULTS}

\section{Colocalization of NudC with Lis1, dynein, and the microtubule organizing center facing the leading pole of cerebellar granule cells with a migrating morphology}

Neonatal cerebellar granule cells have proven useful to study neuronal migration at the cellular level in vitro (Rivas and Hatten, 1995). In this assay, dissociated mouse cerebellar granule cells are allowed to form reaggregate clusters in suspension. Plating the clusters on laminin-coated slides results in the extension of radial neurites on which granule cells migrate as determined by timelapse microscopy (Edmondson and Hatten, 1987; Bix and Clark, 1998). To substantiate the hypothesis that NudC forms a complex with Lis1 and the dynein motor in migrating neurons, P8 granule cell clusters cultured under these conditions were stained pairwise for NudC versus microtubules, CDIC, and Lis1. In cells with a migrating morphology, NudC is localized in neurites and neurite tips (Fig. 1 $A$ ). In the soma, NudC is found polarized on one side of the nucleus (Fig. $1 A, D, G$ ). The polarized fraction of NudC colocalizes with $\alpha$-tubulin, CDIC, and Lis1 (Fig. $1 B, E, H$ ) and is in a region of the soma that also contains the microtubule organizing center (MTOC) (Fig. 1C, F,I). In migrating cerebellar granule cells, the MTOC is rostral to the nucleus and oriented toward the leading pole, in the direction of migration (Rivas and Hatten, 1995). Additionally, the filamentous pattern of NudC, Lis1, and CDIC staining surrounding the nucleus is reminiscent of the reported "cage-like" microtubule network encompassing the soma in migrating cerebellar granule cells (Rivas and Hatten, 1995).

In bipolar (stage III) cerebellar granule cells cultured under conditions in which migration does not occur, NudC immunoreactivity is detected throughout the soma (Fig. $1 J$ ) and overlaps with that of CDIC in a polarized manner on one side of the nucleus (Fig. $1 L$ ) in the region of the MTOC. In E15 cortical neurons cultured under conditions that allow neurite outgrowth, NudC staining occurs at the neurite tips, is diff use throughout the soma, and colocalizes with tubulin at a perinuclear region directly adjacent to the nucleus where the MTOC is located (Fig. 1M,N). These results show that in neurons with stationary morphology, a fraction of cellular NudC localizes to the region of the MTOC, whereas in cells with a migrating morphology, NudC, CDIC, and Lis1 colocalize at the MTOC facing the leading process.

\footnotetext{
of cerebellar granule cells with a migratory morphology. $J-L$, Primary cerebellar granule and glial cells were stained with anti-NudC and anti-CDIC. $J$, NudC immunoreactivity is detected diffusely in the soma (asterisk), neurites, and neurite tips (arrowhead). K, Immunoreactivity against CDIC occurs predominantly in neurites and is highly polarized on one side of the nucleus (arrow). $L$, NudC staining overlaps with CDIC on one side of the nucleus, whereas colocalization is less evident in the rest of the cell. $M, N$, Primary cortical neurons were prepared from E15 rat embryos. NudC immunoreactivity is evident throughout the soma and in neurite tips (arrows). Costaining with antibodies directed against $\beta$ III-tubulin (red) indicates neurite processes. NudC and $\beta$ III-tubulin colocalize (merge; yellow) in a region around the MTOC (arrowhead). Scale bar, $20 \mu \mathrm{m}$.
} 

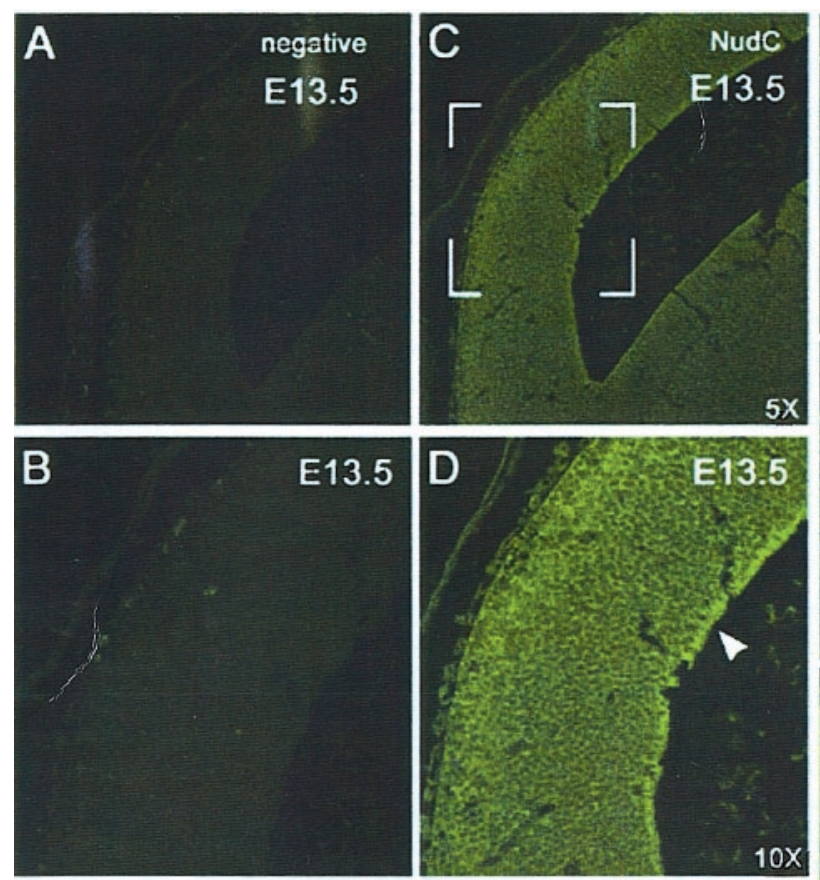

$10 x$
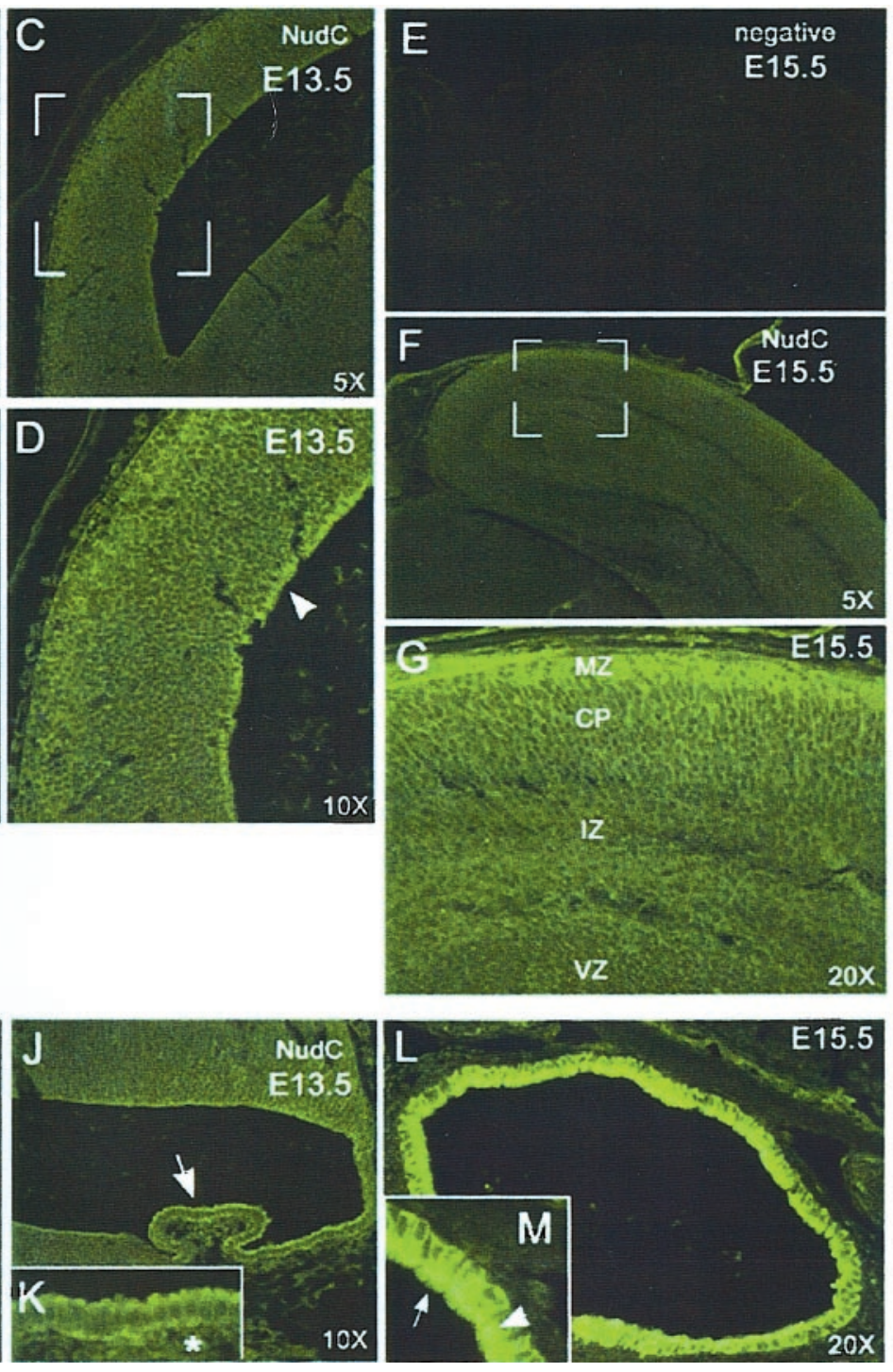
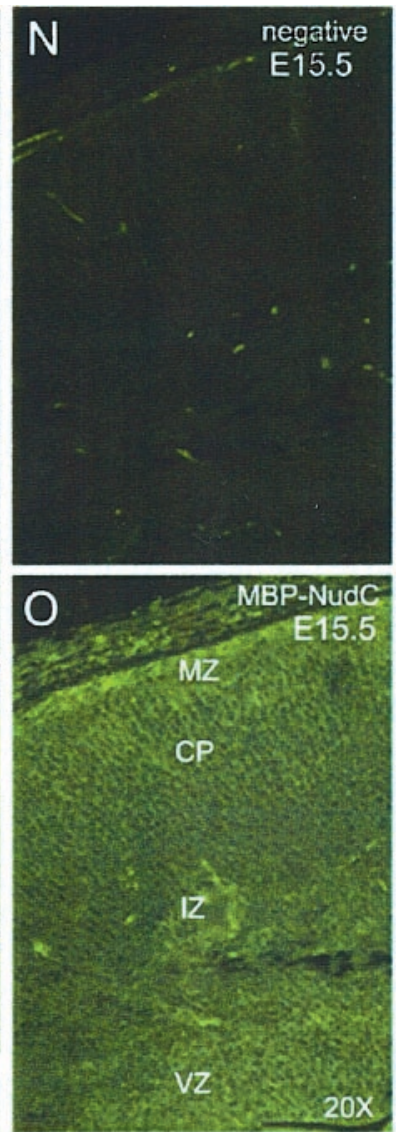

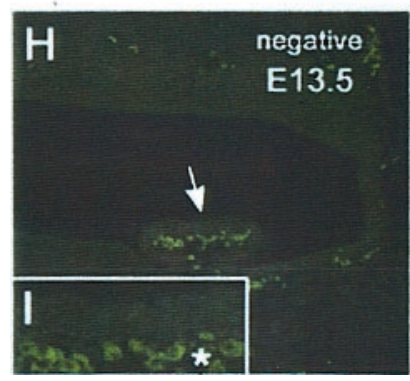

Figure 2. NudC protein expression in the developing mouse brain. $A-D$, Sagittal sections (anterior facing left) through the lateral ventricle of the E13.5 mouse brain. $A$ and $C$ represent adjacent sections. $A, B$, Secondary antibody alone (negative control). $C, D$, Anti-NudC staining (green). NudC immunoreactivity is seen throughout the germinal neuroepithelium and is evident in the ventricular zone (VZ) (arrowhead) in $D$, which corresponds to the boxed area in $C$. E-G, Coronal sections (anterior facing up) through the lateral ventricles of the E15.5 mouse brain. $E, F$, Adjacent sections stained with secondary antibody alone or anti-NudC, respectively. NudC immunoreactivity is seen throughout the neuroepithelium and is high in the marginal zone $M Z$ in $G$, which corresponds to the boxed area in $F . H-K$, Sagittal sections through the fourth ventricle of the E13.5 mouse brain. The choroid plexus (arrow) is stained with secondary antibody alone $(H)$ or with anti-NudC $(J)$. The corresponding enlargements of the upper aspect of the choroid plexus show fluorescent erythrocytes (I, asterisk) within the vasculature of the choroid versus diffuse cytoplasmic staining of NudC $(K)$. $L$, Cross section through the central canal of the E15.5 spinal cord showing high expression of NudC in ependymal cells. $M$, Enlargement of cells in $L$ showing apical enrichment of NudC (arrowhead) beneath the cilia (arrow). A different antibody generated against an MBP-NudC fusion protein was used to stain the E15.5 neocortex. $N$, Secondary antibody alone. $O$, NudC staining using anti-MBP-NudC. Sections were photographed at 5 , 10 , or $20 \times$ magnification as indicated.

\section{NudC is expressed throughout the embryonic neuroepithelium}

We demonstrated previously that $N u d C$ and Lis 1 are coexpressed in embryonic neuroepithelium and that NudC and Lis1 are found as a biochemical complex in brain extracts in vivo (S. M. Morris et al., 1998). Furthermore, we and others have shown that NudC expression is upregulated in proliferating cells and in tissues with high mitotic indices (Morris et al., 1997; Gocke et al., 2000). The nuclei of VZ neuronal progenitors undergo a characteristic oscillatory movement as they progress through the cell cycle (Chenn and McConnell, 1995). We therefore hypothesized that NudC expression would be high in the VZ, where nuclear migration and proliferation are coupled. Immunostaining of E13.5 brain sections with anti-NudC antibodies revealed widespread NudC expression throughout the neuroepithelium of the lateral ventricle (Fig. 2C,D). Additionally, a thin band of immunoreactivity is observed at the $\mathrm{VZ}$ of the lateral ventricle (Fig. 2D). By E15.5, NudC is expressed in the VZ, IZ, and CP but is expressed more abundantly in the MZ of the neocortex (Fig. $2 F, G$ ). To confirm the pattern of NudC staining in the neocortex, an antibody generated against an MBP-NudC fusion protein was used to stain adjacent sections (Fig. 2O). A similar distribution of NudC was observed. The specificity of NudC staining is shown by staining with either secondary antibody and fluorochrome alone (negative 

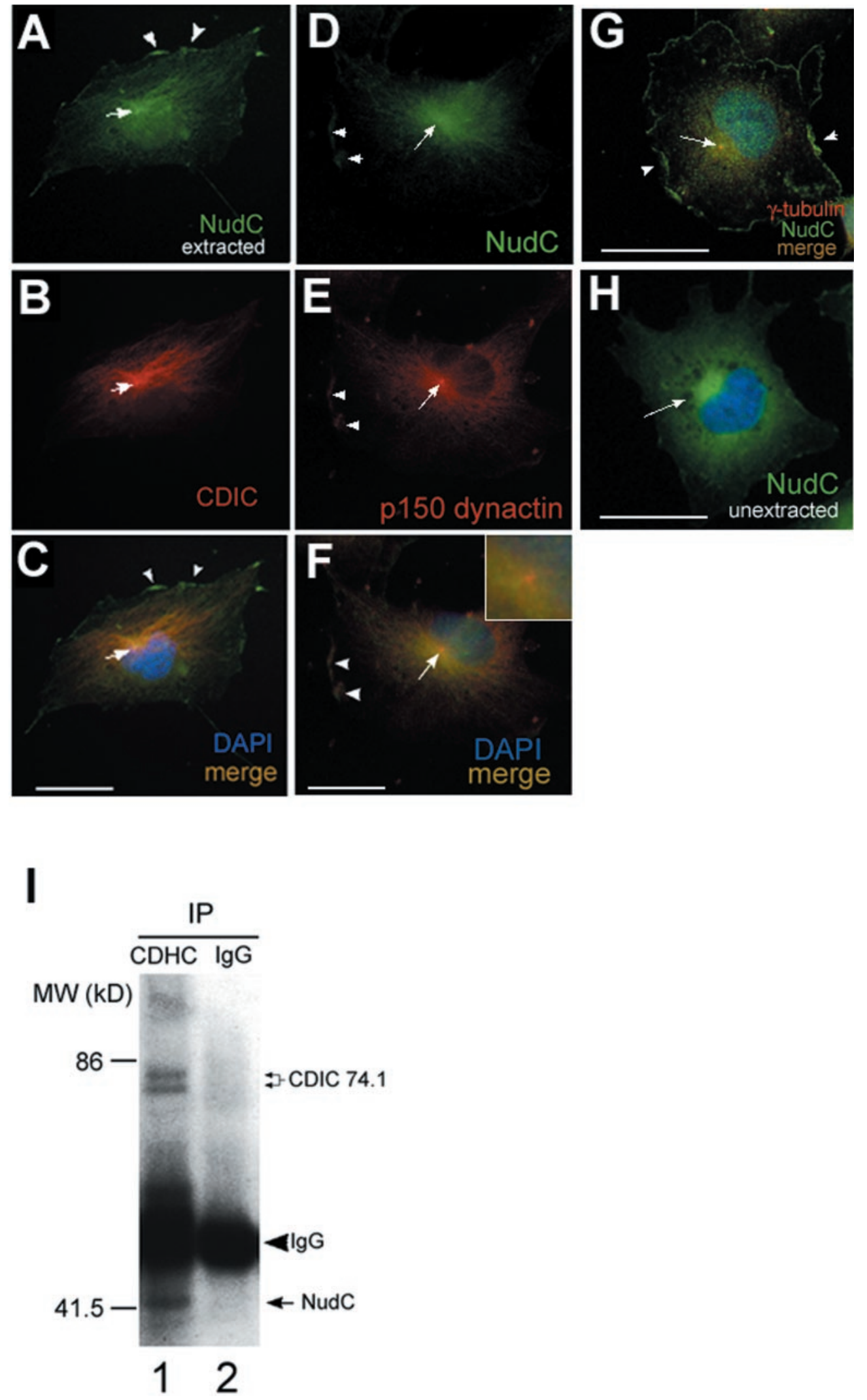

control) (Fig. $1 A, B, E, N)$ or by preincubating anti-NudC with excess immunogen before applying the antibody to the slide. All staining was successfully competed (data not shown). The expression pattern of NudC in the neocortex is similar to that
Figure 3. NudC colocalizes with dynein and dynactin in interphase cells and coimmunoprecipitates with dynein in mouse brain. Fibroblasts were treated briefly with detergent to extract soluble proteins. $A$, Extracted cells show a filamentous staining pattern for $\mathrm{NudC}$ (green) along the microtubule array emanating from the MTOC (arrow), as well as focal sites at the cell periphery (arrowheads). B, Extracted cells stained with anti-CDIC display filamentous pattern emanating from the MTOC (arrow). $C$, NudC staining overlaps with that of CDIC on the interphase microtubule array (arrow) but not at the cell periphery (arrowhead). D, Extracted cells were stained with anti-NudC as in $A$ and costained with anti-p150 dynactin (red) $(E) . F$, Merging NudC and dynactin shows colocalization on interphase microtubules, on peripheral foci (arrowheads), and at the MTOC (arrow, enlargement in top right). $G$, NudC localization at the MTOC (arrow) in COS-1 cells is confirmed by double staining using anti-NudC (green) and anti- $\gamma$ tubulin (red). NudC is again observed at focal sites at the periphery (arrowheads). Nuclei are indicated by DAPI counterstain (blue). $\mathrm{H}$, Unextracted fibroblasts show diffuse cytoplasmic staining with enrichment at the Golgi apparatus (arrow) (Morris and Yu-Lee, 1998). Scale bar, $10 \mu \mathrm{m} . I$, Cytosolic extracts from mouse brain were immunoprecipitated with $5 \mu \mathrm{l}$ anti-CDHC (lane 1) or with $5 \mu \mathrm{g}$ rabbit IgG (lane 2), followed by immunoblotting with both anti-CDIC and anti-NudC. A specific complex is detected among CDHC, CDIC, and NudC (lane 1), but not with rabbit $\operatorname{IgG}$ (lane 2). described for Lis1 and CDIC (Smith et al., 2000). These data indicate that $\mathrm{NudC}$ is expressed at the same developmental stages in the embryonic brain as Lis1 and CDIC and are consistent with our interpretation that NudC plays an important role during 


\section{Direction of migration}

Figure 4. Model for the cooperation of NudC, Lis1, and dynein in mediating nuclear transport in migrating neurons. NudC and Lis1 may be involved in targeting and regulating dynein function at the cell cortex $(A)$ or the interstitial junction $(B)$ at the leading pole of migrating neurons. The minus enddirected activity of dynein is suggested to pull the MTOC and the associated nucleus in the direction of migration. NudC, Lis1, and dynein may also be involved in tethering the nucleus to the MTOC and transporting it along microtubules $(C)$.

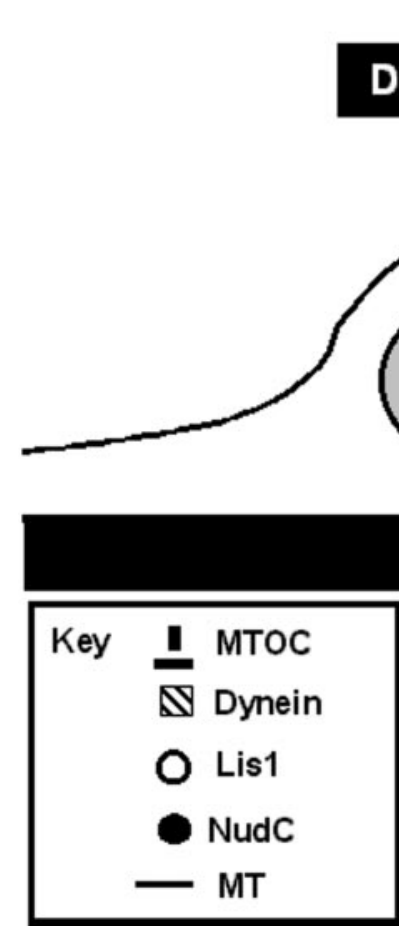

Migration substrate neurogenesis in the $\mathrm{VZ}$ and in neuronal migration in the $\mathrm{IZ}$ and $\mathrm{CP}$.

\section{NudC is expressed in the choroid plexus and in ependymal cells}

Two additional areas of the developing brain show NudC immunoreactivity, including the choroid plexus (Fig. 2J,K) and ependymal cells lining the central canal of the spinal cord (Fig. $2 L, M)$. The choroid plexus is composed of columnar epithelium rich in microvilli and is responsible for the production and secretion of cerebrospinal fluid. NudC expression in the choroid plexus agrees with data reported by others (Gocke et al., 2000) and with our previous suggestion that NudC is involved in polarized cell function and secretion (Morris and Yu-Lee, 1998). Ciliated ependymal cells are responsible for circulating cerebrospinal fluid in the ventricles and central canal of the CNS. NudC is highly expressed in ependymal cells and is enriched at the apical aspect of the cell (Fig. 2M), where NudC may be involved in ciliary motility (Gocke et al., 2000). Interestingly, Lis1 is also expressed in human embryonic ependymal cells (Clark et al., 1997).

\section{NudC colocalizes with dynein/dynactin on the interphase microtubule array}

To further examine the subcellular localization of NudC in relation to dynein, fibroblasts were treated briefly with detergent to extract soluble proteins. NudC and CDIC stain brightly in a filamentous pattern adjacent to the nucleus and at the MTOC (Fig. $3 A-C$ ). Similarly, NudC colocalizes with the dynein regulator, p150 dynactin, on the microtubule array and at the MTOC (Fig. 3D-F). The presence of NudC at the MTOC was confirmed by colocalizing NudC with $\gamma$-tubulin (Fig. 3G). An unextracted cell (Fig. $3 H$ ) shows the characteristic cytosolic and predominant Golgi staining as reported previously (Morris and Yu-Lee, 1998). Interestingly, foci of NudC staining remain at the cell cortex after detergent extraction (Fig. 3A,D,G), suggesting that a portion of cellular NudC is associated with the cortical cytoskeleton. Only p150 dynactin but not CDIC colocalized with NudC at focal sites
(Fig. 3, compare $C, F$, arrowheads). These data provide the first evidence at the subcellular level that NudC colocalizes with components of the dynein motor and microtubules in neurons and fibroblasts and that NudC is found at the MTOC as well as at discrete foci at the cell periphery.

\section{NudC immunoprecipitates with dynein components}

To confirm that a biochemical interaction occurs between NudC and components of the dynein molecular motor, we performed coimmunoprecipitation assays using mouse brain extracts. Both CDIC and NudC are specifically found in the CDHC immunoprecipitate (Fig. 3I, lane 1) and not in samples immunoprecipitated with unrelated antibodies (IgG) (Fig. 3I, lane 2). This result demonstrates that a NudC/dynein complex can be detected in vivo.

\section{DISCUSSION}

Nuclear translocation and neuronal migration are two closely linked cellular events in the developing neocortex. We showed previously that two genes involved in nuclear movement and neuronal migration, $N u d C$ and Lis1, respectively, are coexpressed throughout the developing neuroepithelium by in situ hybridization (S. M. Morris et al., 1998). By examining endogenous protein expression in E13.5-E15.5 brain, we showed that NudC is highly expressed in neuronal progenitors of the $\mathrm{VZ}$, in migrating $\mathrm{IZ}$ and $\mathrm{CP}$ neurons, and in the MZ (Fig. $2 C, D, F, G, O$ ). The expression profile of NudC overlaps with that of Lis1 and CDIC, which are highly expressed in neurons in the $\mathrm{CP}$ and $\mathrm{MZ}$ (Sasaki et al., 2000; Smith et al., 2000). Recent reports also show that Lis1 interacts with dynein (Faulkner et al., 2000; Smith et al., 2000), NudE (Feng et al., 2000), and NudeL (Niethammer et al., 2000; Sasaki et al., 2000). Together, these observations show that the nud genes, which are genetically linked in a nuclear movement pathway in Aspergillus, encode proteins that function as a biochemical complex in multiple cell types and may play an important role during brain development. Interestingly, in addition to 
neuronal migration, Lis1 is suggested to play a role in neuroblast proliferation (Liu et al., 2000) and in spindle orientation during cell division (Dawe et al., 2001; Faulkner et al., 2000), whereas NudC expression is observed in proliferating neuronal progenitors (this study), in cells induced to proliferate (Morris and Yu-Lee, 1998), and in tissues with high proliferative indices (Gocke et al., 2000).

Functionally, Lis1 localizes to the cortex of Drosophila oocytes where it has been shown to act as a cortical anchor for dynein (Swan et al., 1999). Similarly, NudC is highly enriched at the cell cortex of amphibian oocytes (Moreau et al., 2001). In Caenorhabditis elegans blastomeres, dynactin is suggested to anchor dynein to cortical sites, and through the minus end-directed activity of dynein acting on astral microtubules, the associated centrosome and nucleus are transported to the cell cortex (Waddle et al., 1994; Reinsch and Gonczy, 1998). Recent studies also show that Lis1 potentiates dynein/dynactin activity in a minus end-directed manner toward the centrosome, influencing the organization of microtubules as well as the distribution of the Golgi complex (Smith et al., 2000; Wynshaw-Boris and Gambello, 2001). We envision an analogous mechanism for nuclear transport in migrating neurons (Fig. 4), in which NudC and Lis1 anchor dynein along polarized microtubules at the leading pole, either at the cell cortex or along the interstitial junction between the migrating granule cell and the substratum (Rivas and Hatten, 1995). By virtue of the minus end-directed movement of tethered dynein, the nucleus and its associated centrosome are actively pulled through the leading process. Furthermore, the colocalization of NudC, Lis1, dynein, and microtubules around the nucleus of migrating cerebellar granule cells proximal to the MTOC (Fig. 1) suggests that the complex may also be involved in tethering the nucleus to the centrosome, thus aiding in nuclear transport.

The precise mechanism of NudC action in neuronal migration as well as in polarized, secretory, or proliferating cells is unknown. However, given the important role for dynein in vesicular transport and in chromosomal attachment to mitotic spindles during mitosis, and the recent demonstration that Lis1 regulates dynein assembly and function (Smith et al., 2000; Vallee et al., 2001; Wynshaw-Boris and Gambello, 2001), we suggest that NudC is a regulatory component of the dynein motor complex. Our findings therefore offer new insights into the molecular functions of NudC and provide a framework from which to test the function of NudC as a novel component of the Lis1/dynein motor complex involved in neurogenesis, neuronal migration, and brain development.

\section{REFERENCES}

Bix GJ, Clark GD (1998) Platelet-activating factor receptor stimulation disrupts neuronal migration in vitro. J Neurosci 18:307-318.

Chenn A, McConnell SK (1995) Cleavage orientation and the asymmetric inheritance of notch1 immunoreactivity in mammalian neurogenesis. Cell 82:631-641.

Chiu Y-H, Xiang X, Dawe AL, Morris NR (1997) Deletion of nudC, a nuclear migration gene of Aspergillus nidulans, causes morphological and cell wall abnormalities and is lethal. Mol Biol Cell 8:1735-1749.

Clark GD, Mizuguchi M, Antalffy B, Barnes J, Armstrong D (1997) Predominant localization of the LIS family of gene products to Cajal- retzius cells and ventricular neuroepithelium in the developing human cortex. J Neuropathol Exp Neurol 56:1044-1052.

Dawe AL, Caldwell KA, Harris PM, Morris NR, Caldwell GA (2001) Evolutionarily conserved nuclear migration genes are neuronally expressed and required for early embryonic development in C. elegans. Dev Genes Evol 211:434-441.

Edmondson JC, Hatten ME (1987) Glial-guided granule neuron migration in vitro: a high-resolution time-lapse video microscopic study. J Neurosci 7:1928-1934

Efimov VP, Morris NR (2000) The LIS1-related NUDF protein of Aspergillus nidulans interacts with the coiled-coil domain of the NUDE/ RO11 protein. J Cell Biol 150:681-688.

Faulkner NE, Dujardin DL, Tai C-Y, Vaughan KT, O'Connell CB, Wang Y-L, Vallee RB (2000) A role for the lissencephaly gene LIS1 in mitosis and cytoplasmic dynein function. Nat Cell Biol 2:784-791.

Feng Y, Walsh CA (2001) Protein-protein interactions, cytoskeletal regulation and neuronal migration. Nat Rev Neurosci 2:408-416.

Feng Y, Olson EC, Stukenberg PT, Flanagan LA, Kirschner MW, Walsh CA (2000) Lis1 regulates CNS lamination by interacting with mNudE, a central component of the centrosome. Neuron 28:665-679.

Gocke CD, Osmani SA, Miller BA (2000) The human homologue of the Aspergillus nuclear migration gene nudC is preferentially expressed in dividing cells and ciliated epithelia. Histochem Cell Biol 114:293-301.

Liu Z, Steward R, Luo L (2000) Drosophila Lis1 is required for neuroblast proliferation, dendritic elaboration and axonal transport. Nat Cell Biol 2:776-783.

Moreau N, Aumais JP, Prudhomme C, Morris SM, Yu-Lee L (2001) NudC expression during amphibian development. Int $\mathrm{J}$ Dev Biol, in press.

Morris NR (2000) Nuclear migration: from fungi to the mammalian brain. J Cell Biol 148:1097-1101.

Morris NR, Efimov VP, Xiang X (1998) Nuclear migration, nucleokinesis and lissencephaly. Trends Cell Biol 8:467-470.

Morris SM, Yu-Lee L (1998) Expression of RNUDC, a potential nuclear movement protein, in mammalian cells: localization to the Golgi apparatus. Exp Cell Res 238:23-32.

Morris SM, Anaya P, Xiang X, Morris NR, May GS, Yu-Lee L (1997) A prolactin-inducible $\mathrm{T}$ cell gene product is structurally similar to the Aspergillus nidulans nuclear movement protein NUDC. Mol Endocrinol 11:229-236.

Morris SM, Albrecht U, Reiner O, Eichele G, Yu-Lee L (1998) The lissencephaly gene product LIS1, a protein involved in neuronal migration, interacts with a nuclear movement protein NUDC. Curr Biol 8:603-606.

Niethammer M, Smith DS, Ayala R, Peng J, Ko J, Lee M-S, Morabito M, Tsai L-H (2000) NudEL is a novel cdk5 substrate that associates with Lis1 and cytoplasmic dynein. Neuron 28:697-711.

Osmani AH, Osmani SA, Morris NR (1990) The molecular cloning and identification of a gene product specifically required for nuclear movement in Aspergillus nidulans. J Cell Biol 111:543-551.

Reiner O, Carrozzo R, Shen Y, Wehnert M, Faustinella F, Dobyns DW, Caskey CT, Ledbetter DH (1993) Isolation of a Miller-Dieker lissencephaly gene containing $G$ protein $\beta$-subunit-like repeats. Nature 364:717-721.

Reinsch S, Gonczy P (1998) Mechanisms of nuclear positioning. J Cell Sci 111:2283-2295.

Rivas RJ, Hatten ME (1995) Motility and cytoskeletal organization of migrating cerebellar granule neurons. J Neurosci 15:981-989.

Sasaki S, Shionoya A, Ishida M, Gambello MJ, Yingling J, WynshawBoris A, Hirotsune S (2000) A Lis1/NudEL/cytoplasmic dynein heavy chain complex in the developing and adult nervous system. Neuron 28:681-696.

Smith DS, Niethammer M, Ayala R, Zhou Y, Gambello MJ, WynshawBoris A, Tsai L-H (2000) Regulation of cytoplasmic dynein behaviour and microtubule organization by mammalian Lis1. Nat Cell Biol 2:767-775.

Swan A, Nguyen T, Suter B (1999) Drosophila lissencephaly-1 functions with Bic-D and dynein in oocyte determination and nuclear positioning. Nat Cell Biol 1:444-448.

Vallee RB, Tai C-Y, Faulkner NE (2001) LIS1: cellular function of a disease-causing gene. Trends Cell Biol 11:155-160.

Waddle JA, Cooper JA, Waterston RH (1994) Transient localized accumulation of actin in Caenorhabditis elegans blastomeres with oriented asymmetric divisions. Development 120:2317-2328.

Wynshaw-Boris A, Gambello MJ (2001) LIS1 and dynein motor function in neuronal migration and development. Genes Dev 15:639-651. 